\title{
Inventario de Ansiedad y Depresión Hospitalaria y Escala de Retiro de Patrón de Conducta tipo A en pacientes con enfermedades cardiovasculares: propiedades psicométricas
}

\author{
Inventory of Anxiety and Hospital Depression and Type A \\ Behavior Pattern Withdrawal Scale in a population of patients \\ with cardiovascular diseases: Psychometric properties
}

\author{
Bibian Herrera Navarrete ${ }^{1}$, Oscar Galindo Vázquez $z^{2,3}$ \\ y Abel Lerma Talamantes ${ }^{4}$
}

\begin{abstract}
RESUMEN
Introducción: La sintomatología de ansiedad, depresión y conducta tipo A son aspectos psicológicos prevalentes en población con enfermedades cardiovasculares. Objetivo: validar el Inventario de Ansiedad y Depresión (HADS) y la Escala de Retiro de Patrón de Conducta tipo A (ERCTA) en una población de pacientes con enfermedades cardiovasculares. Método: Se empleó un diseño transversal, con un muestreo no probabilístico, por disponibilidad. Se incluyó a 200 pacientes diagnosticados con enfermedades cardiovasculares, con una media de edad de 59.7 años, de los cuales 59\% eran hombres. Los criterios de inclusión fueron ser pacientes con enfermedades cardiovasculares con diagnóstico médico confirmado, en cualquier tratamiento médico, y saber leer y escribir. Los criterios de exclusión fueron sufrir limitaciones visuales o auditivas severas y afectaciones cognitivas, y como criterios de eliminación no concluir la investigación o llenar incorrectamente los cuestionarios. Resultados: El análisis factorial del HADS identificó un solo factor, con un coeficiente alfa de la escala global de 0.94 y una varianza explicada de 59.6\%. La estructura factorial del ERCTA, ajustada a un solo factor, mostró un coeficiente alfa de la escala global de 0.93 y una varianza explicada de $67.3 \%$. La validez concurrente entre ambos instrumentos por medio de correlación mostró resultados significativos. Discusión: Los inventarios HADS y ERCTA mostraron ser instrumentos válidos y confiables para su uso en la atención clínica y la investigación de pacientes con enfermedades cardiovasculares. Esta población puede requerir atención psicosocial, además de la que se le brinde a su salud.
\end{abstract}

Palabras clave: Ansiedad; Depresión; Enfermedades cardiovasculares; Patrón de conducta tipo A; Validación de pruebas.

\footnotetext{
${ }^{1}$ Universidad Latina, Campus Sur, Av. Cuauhtémoc No. 1614, Col. San Lorenzo Tezonco, Deleg. Iztapalapa, 09900 Ciudad de México, tel. (55)13-12-47-08, correo electrónico: b.herreranavarrete@gmail.com. Artículo recibido el1 de agosto y aceptado el 15 de septiembre de 2017. ${ }^{2}$ Instituto Nacional de Cancerología, Servicio de Psicooncología, Av. San Fernando 22, Col. Sección Xvi, Delegación Tlalpan, 14080 Ciudad de México, tel. (55)56-28-04-00.

${ }^{3}$ Facultad de Psicología, Universidad Nacional Autónoma de México, Av. Universidad 3004, Col. Copilco Universidad, Del. Coyoacán, 04510 Ciudad de México.

${ }^{4}$ Escuela de Enfermería, Instituto Nacional de Cardiología "Ignacio Chávez", Belisario Domínguez, Sección 16, Belisario Domínguez, Secc. 16, Del. Tlalpan, 14080 Ciudad de México.
} 


\begin{abstract}
Background: Anxiety and depression symptoms as well as type A behavior are prevalent psychological conditions in people with cardiovascular disease. $\mathrm{Ob}-$ jective: to validate the Hospital Anxiety and Depression Scale (HADS) and the Retirement Scale Pattern A (ERCTA) in a population of patients with cardiovascular disease. Method: A cross-sectional design was used, and participants were recruited through a non-probabilistic procedure on the basis of availability. A total of 200 patients diagnosed with cardiovascular disease, with an average of 59.7 years of age participated (59\% were men). Participants were selected if they had confirmed cardiovascular disease diagnosis, being in any type of medical treatment, and fully literate. Potential participants were excluded if they had serious uncorrected visual or hearing limitations. Data from participants who did not complete the study's protocol or improperly filled out the corresponding questionnaires were excluded from the analyses. Results. Factor analysis of the HADS revealed a single factor and yielded a 0.947 Cronbach's alpha coefficient and a 59.6\% explained variance. The factorial structure of ERCTA, adjusted to a single factor, showed an alpha coefficient of 0.930 in the global scale, and an explained variance of $67.3 \%$. Concurrent validity between the two instruments showed a statistically significant correlation. Discussion: The HADS and ERCTA inventories were shown to be valid and reliable instruments for their use in clinical and research endeavors with cardiovascular diseased persons. This population may require psychosocial management in addition to their medical health care.
\end{abstract}

Key words: Anxiety; Depression; Cardiovascular disease; Type A behavior pattern; Test validation.

\section{INTRODUCCIÓN}

$\mathrm{S}$ egún el Sistema Nacional de Información en Salud (s/f), las enfermedades cardiovasculares (ECV), aunque se establecen de forma lenta y subclínica durante décadas, se manifiestan la mayoría de las veces de manera abrupta e imprevista (Achiong, Achiong y Achiong, 2015). Dichas enfermedades se deben a trastornos del corazón y de los vasos sanguíneos, como las cardiopatías coronarias (ataques cardíacos), las enfermedades cerebrovasculares (apoplejía), el aumento de la tensión arterial (hipertensión), las vasculopatías periféricas, las cardiopatías reumáticas y congénitas y la insuficiencia cardíaca (Organización Mundial de la Salud [OMS], 2015).

Las ECV son la principal causa de muerte en todo el mundo. En 2012 murieron 17.5 millones de personas por esta causa, lo que representa $31 \%$ de todas las muertes registradas en el mundo. De estas, 7.4 millones se debieron a cardiopatía coronaria y 6.7 millones a accidentes cerebrovasculares (OMS, 2015). En México, en 2015 hubo 128,731 defunciones debido a enfermedades del corazón, lo que las convierte en la primera causa de muerte (Instituto Nacional de Estadística y Geografía, 2016).

En las personas con ECV o con alto riesgo cardiovascular debido a la presencia de uno o más factores de riesgo, como la hipertensión arterial, la diabetes, la hiperlipidemia o alguna ECV ya confirmada, son fundamentales la detección precoz y el tratamiento temprano por medio de servicios de orientación o la administración de fármacos, según corresponda (OMS, 2015).

Durante décadas, las ECV han sido asimismo la principal causa de muerte y discapacidad en los países occidentales, pero con la mejora de tratamientos como la angioplastía y los avances del tratamiento médico, ha empezado a reducirse su impacto hasta quedar por debajo del cáncer en cuanto a la mortalidad asociada; sin embargo, en personas de más de 65 años la primera causa de muerte sigue siendo la cardiopatía, lo que plantea dificultades considerables para su diagnóstico y tratamiento (Roger, Lloyd-Jones, Adams, Brown et al., 2010).

Con el aumento de las enfermedades cardiovasculares ha habido un interés también creciente en examinar la asociación entre una pobre salud cardiovascular y el bienestar psicológico (Tuckey, Dollard, Saebel y Berry, 2010). De acuerdo a Yohannes, Willgoss, Baldwin y Connolly (2010), en esta población las dos comorbilidades más comunes y menos tratadas son la depresión y la ansiedad, a pesar de que tienen un impacto central en la función psicológica y la interacción social de las personas con enfermedades cardiovasculares. Adicionalmente, se ha identificado el patrón de conducta tipo A como un factor del comportamiento de riesgo en este grupo de pacientes. 


\section{Depresión y ansiedad en pacientes con enfermedad cardiovascular}

De todos los factores psicológicos, la depresión ha sido objeto de gran atención por parte de los investigadores durante la última década (American Psychiatric Association, 2014). Las primeras investigaciones sobre la relación entre depresión y ECV se debieron en gran parte a algunas observaciones que indicaban que la depresión diagnosticada durante la hospitalización debida a una ECV se asociaba con un aumento significativo del riesgo de muerte en el año siguiente (Frasure-Smith, Lespérance y Talajic, 1993). De hecho, dado el abrumador número de estudios que relacionan la depresión con la ECV, las recomendaciones clínicas recientes incluyen, como un elemento central de la asistencia estándar (Lichtman et al., 2008), la detección sistemática de tal condición y su tratamiento en los pacientes cardiacos.

En efecto, la depresión se encuentra frecuentemente en estos pacientes y se asocia a un mayor riesgo de mortalidad, lo que puede aumentar en ellos el riesgo de sufrir eventos cardiacos recurrentes y, como consecuencia, morir al no adherirse al tratamiento farmacológico y por el cambio negativo de los comportamientos relacionados con el estilo de vida (Lane, Chong y Lip, 2006). La depresión es asimismo tres veces más frecuente en los pacientes que han sufrido un infarto agudo al miocardio que en la población general (Lichtman et al., 2008; Thombs, 2006). En los individuos con cardiopatía es frecuente la depresión, en especial tras sufrir un infarto al miocardio; de hecho, al menos uno de cada cinco pacientes cumplen esos criterios diagnósticos (Thombs et al., 2008).

Por su parte, la ansiedad se ha asociado también a un aumento de la mortalidad cardiaca, aunque las observaciones al respecto son mucho menos consistentes y parecen depender en parte de la gravedad de la ECV (Smith y Blumenthal, 2011). La ansiedad tiene diversas consecuencias médicas y psicológicas en pacientes con alguna enfermedad cardiaca -en especial cuando aquella es persistente o grave-, e incluso dificulta la adherencia a los tratamientos prescritos por el médico y los cambios en el estilo de vida recomendados; no llevar a cabo dichas modificaciones aumenta el riesgo de sufrir eventos cardiacos agudos y de tener complicaciones en el hospital después de un síndrome coronario agudo (Moser, 2007).

\section{Patrón de conducta tipo A}

De acuerdo con Evans (1991), las personas con un patrón de conducta tipo A (PCTA en lo sucesivo) muestran un elevado afán competitivo, de logro y reconocimiento, con tendencia a la hostilidad y la agresión, así como impaciencia y una sensación extrema de urgencia en el tiempo. Una característica central de este patrón de conducta, perjudicial para la salud de las personas que lo manifiestan, es el peculiar estilo de afrontamiento basado en un uso abusivo de estrategias activas que se acompañan de respuestas emocionales de hostilidad y apresuramiento (Palmero et al., 2007).

Desde hace más de una década, el PCTA se considera como un claro indicador de riesgo coronario (Mittleman et al., 1995; Muller, Adela, Nesto y Tofler, 1994; Willich, Maclure, Mittleman, Arntz y Muller, 1993). Diversos investigadores (Barefoot, Larsen, Bonderleith y Schroll, 1995; Everson et al., 1997; Kawachi, Sparrow, Spiro, Vokonas y Weiss et al., 1996) han mostrado que los altos niveles de ira y hostilidad se relacionan de un modo importante con el aumento en la morbilidad debida a ECV.

El PCTA está configurado por componentes formales (voz alta, habla rápida, actividad psicomotora y gesticulación excesivas, tensión de la musculatura facial y otros manierismos típicos); actitudes y emociones (hostilidad, impaciencia, ira y agresividad); aspectos motivacionales (motivación de logro, competitividad, orientación al éxito, afán de superación y ambición); conductas abiertas o manifiestas (urgencia de tiempo, prisa, rapidez, hiperactividad e implicación en el trabajo), y aspectos cognitivos (necesidad de control ambiental y estilo atribucional característico) (Friedman y Rosenman, 1974).

El PCTA se ha convertido en una parte importante en el desarrollo de EVC en virtud de que el daño arterial se produce debido a que quienes tienen este patrón de conducta responden habitualmente a todas las demandas del entorno, lo que aumenta la actividad del sistema nervioso simpático de tal modo que su constante sobrecarga de actividad puede precipitar un evento cardiovascu- 
lar. Se ha observado que los hombres sufren más infartos que las mujeres, pero cuando ellas los llegan a tener, son invariablemente mujeres con conducta tipo A (Friedman, 1996).

Se han desarrollado diversos instrumentos para evaluar los síntomas de depresión y ansiedad en pacientes médicamente enfermos. Uno de ellos es la Escala Hospitalaria de Ansiedad y Depresión (HADS por sus siglas en inglés), desarrollada por Zigmond y Snaith (1983), que es un instrumento autoaplicado, lo que permite utilizarlo en medios hospitalarios no psiquiátrico o en la atención primaria. Este instrumento de catorce reactivos considera dimensiones cognitivas y afectivas y omite aspectos somáticos (insomnio, fatiga, pérdida de apetito), con lo que evita atribuirlos a la enfermedad. La escala original ha sido validada en población mexicana aplicándola a sujetos obesos (López et al., 2000), mujeres en puerperio complicado (Villegas, 2004), pacientes con infección por VIH (Nogueda, Pérez, Barrientos, Robles y Sierra, 2013), pacientes con cáncer (Galindo, Benjet et al., 2015) y cáncer de mama en particular (Ornelas, Tufiño, Sánchez Sosa, 2011), personas con trastornos de la conducta alimentaria (Barriguete et al., 2017) y cuidadores primarios informales de pacientes con cáncer (Galindo, Meneses et al., 2015). Sin embargo, se carece de una versión validada para población mexicana con enfermedad cardiovascular.

En relación con el PCTA, se han desarrollado diversas escalas, entre las cuales están la Bortner Rating Scale (Bortner, 1969), la Framingham Type A Scale) (Haynes, Levin, Scotch, Feinleib y Kannel, 1978) y la Jenkins Activity Survey (Jenkins, Rosenman y Zyzanski, 1979). Estas escalas se caracterizan por tener reactivos con propiedades psicométricas adecuadas; no obstante, el número de estos difículta su empleo cotidiano en procedimientos clínicos de tamizaje. Por ello, la Escala de Retiro de Patrón de Conducta Tipo A (ERCTA en lo sucesivo), cuyo objetivo principal es la detección (screening), representa una medida breve, con un número reducido de ítems (ocho) y un tiempo de aplicación de alrededor de cinco minutos.

Así, el objetivo de este trabajo fue obtener las propiedades psicométricas del Inventario de
Ansiedad y Depresión (HADS) y la Escala de Retiro de Patrón de Conducta tipo A (ERCTA) en una población de pacientes con enfermedades cardiovasculares.

\section{MÉTODO}

\section{Participantes}

Se estudió una muestra no probabilística de 200 pacientes de ambos sexos, con un promedio de 59.7 años, que acudían a consulta en dos instituciones públicas mexicanas del tercer nivel de atención hospitalaria. En la Tabla 1 se muestran las características de los participantes.

\section{Procedimiento}

La obtención de los participantes fue por disponibilidad durante las visitas que hicieron los investigadores a los servicios ambulatorios de dos clínicas de segundo nivel de atención pública y privada a lo largo de un año. Mediante la firma de un formato de consentimiento informado, cada uno de los participantes aceptó participar en el presente estudio una vez que le fue explicado adecuadamente.

Los criterios de inclusión fueron ser pacientes con enfermedades cardiovasculares con diagnóstico médico confirmado, estar en cualquier tratamiento médico y saber leer y escribir. Las limitaciones visuales o auditivas severas y las afectaciones cognitivas se consideraron como criterios de exclusión, y como criterio de eliminación no concluir la investigación o llenar incorrectamente los cuestionarios.

\section{Instrumentos}

\section{Cédula de identificación}

Se diseñó una cédula de identificación del participante que incluía datos sociodemográficos y clínicos, tales como edad, sexo, nivel de estudios, estado civil, diagnóstico, tratamiento médico y tiempo transcurrido desde el diagnóstico. 
Tabla 1. Características de la muestra de pacientes con enfermedades cardiovasculares $(\mathrm{N}=200)$.

\begin{tabular}{|c|c|c|c|c|c|}
\hline & f & $\%$ & & f & $\%$ \\
\hline \multicolumn{3}{|c|}{ Género } & \multicolumn{3}{|l|}{ Diagnóstico } \\
\hline Masculino & 118 & 59.0 & Hipertensión & 118 & 58.0 \\
\hline Femenino & 82 & 41.0 & Cardiopatía isquémica & 27 & 13.5 \\
\hline \multicolumn{3}{|c|}{ Escolaridad } & Arritmia & 5 & 2.5 \\
\hline Ninguna & 5 & 2.5 & Sincope vasovagal & 8 & 4.0 \\
\hline Primaria & 14 & 7.0 & Cardiopatía hipertensiva & 1 & 0.5 \\
\hline Secundaria & 22 & 11.0 & Insuficiencia aórtica & 2 & 1.0 \\
\hline Bachillerato & 56 & 28.0 & Insuficiencia cardiaca & 20 & 10.0 \\
\hline Licenciatura & 84 & 42.0 & Angina de pecho & 10 & 5.0 \\
\hline Posgrado & 19 & 9.5 & Taquicardia supraventricular & 6 & 3.0 \\
\hline \multicolumn{3}{|c|}{ Estado civil } & Prótesis valvular aórtica & 3 & 1.5 \\
\hline Soltero & 9 & 4.5 & \multicolumn{3}{|c|}{ Enfermedad comórbida } \\
\hline Casado & 135 & 67.5 & Sí & 142 & 71.0 \\
\hline Separado & 32 & 16.0 & No & 58 & 29.0 \\
\hline Viudo & 24 & 12.0 & \multicolumn{3}{|c|}{ Atención en salud mental } \\
\hline \multicolumn{3}{|c|}{ Consumo de tabaco } & Sí & 7 & 3.5 \\
\hline Sí & 79 & 39.5 & No & 193 & 96.5 \\
\hline No & 121 & 60.5 & \multicolumn{3}{|c|}{ Familiares con enfermedad cardiovascular } \\
\hline \multirow{2}{*}{\multicolumn{3}{|c|}{ Edad (rango): $31-88$ años }} & Sí & 143 & 71.5 \\
\hline & & & No & 57 & 28.5 \\
\hline
\end{tabular}

Escala Hospitalaria de Ansiedad y Depresión (HADS) (Zigmond y Snaith (1983).

Dicha escala es un instrumento autoaplicado que se utiliza en medios hospitalarios no psiquiátricos y en atención primaria y que está conformado por 14 reactivos. Cada reactivo tiene cuatro posibles respuestas que puntúan de 0 a 3 , para un total de 0 a 21. Tanto en la ansiedad como en la depresión, se considera normal la puntuación de 0 a 7 ; como dudosa la que va de 8 a 10, y como un problema clínico la de 11 o más. Diversos estudios reportan tasas de respuesta de 95 a 100\%. La escala puede responderse entre 5 y 6 minutos; los pacientes con enfermedades crónicas degenerativas pueden responderla fácilmente y el equipo de salud aplicarla de igual manera (Bjelland, Dahl, Haug y Neckelmann, 2002). La escala original ha sido validada en población mexicana en diferentes grupos de pacientes (Barriguete, Pérez, De la Vega, Barriguete y Rojo, 2017; Galindo, Meneses et al., 2015; López et al., 2000; Nogueda et al., 2013; Ornelas, Tufiño y Sánchez-Sosa, 2011; Villegas, 2004) y en cuidadores primarios informales (Galindo et al., 2015), reportándose que cuenta con propiedades psicométricas adecuadas y un coeficiente alfa de Cronbach que varía entre 0.78 y 0.90 .

Escala de Retiro de Patrón de Conducta tipo A (ERCTA) (Rodríguez, Gil y Martínez, 1996).

Esta escala es autoaplicable y se compone de ocho ítems con formato de respuesta que va de 1 a 5 puntos, con un coeficiente de confiabilidad alfa de Cronbach de 0.74 , considerado alto por sus autores dado su reducido número de ítems. Tiene dos factores: Orientación positiva al trabajo y Tensión laboral o aspectos negativos en el trabajo.

\section{Procedimiento}

El piloteo y adaptación de los instrumentos HADS y el ERCTA se llevaron a cabo en una muestra de veinte pacientes con cualquier diagnóstico cardiovascular. De acuerdo a los lineamientos internacionales (Koller et al., 2007), para evaluar la dificultad o una posible confusión en los reactivos se consideró la manera en que la pregunta estaba formulada o si resultaba ofensiva; en su caso, se preguntó a los participantes cómo podrían enun- 
ciarla. También se evaluaron la comprensión de las instrucciones y las opciones de respuesta.

Todos los cuestionarios se capturaron y organizaron en una base de datos para su correspondiente análisis mediante el paquete estadístico SPSS de Windows, versión 20. Se hizo un análisis descriptivo de cada reactivo para asegurar que no hubiera datos extremos y que contara con ellos en todas las casillas resultantes. Se generó una nueva variable con la suma de todos los reactivos y se identificaron los cuartiles extremos, con los cuales se corrió una prueba $t$ de Student para dos muestras independientes, donde se buscó que hubiera significancia en todos los reactivos, demostrando con ello que podían discriminar entre tener o no tener el constructo medido.

Después se corrió un análisis cruzado con todos los reactivos para confirmar que las puntuaciones de los extremos cuartilares identificados corrieran en direcciones opuestas. Finalmente, se identificó la estructura factorial mediante un análisis factorial de componentes principales con ro- tación varimax. La relación entre la escala HADS y el ERCTA se analizó mediante el coeficiente de correlación de Pearson, y la consistencia interna con el coeficiente alfa de Cronbach.

\section{RESULTADOS}

El análisis de confiabilidad de la escala HADS obtuvo un coeficiente alfa de Cronbach total de 0.947. El valor de la prueba Káiser-Meyer-Olkin (KMO) fue de 0.953 ( $\mathrm{p}=0.001)$, lo que confirma que la muestra utilizada fue adecuada para este análisis. Debido a que las correlaciones internas iban de medias a bajas, el análisis factorial se hizo mediante rotación varimax, ajustándose a un factor que comprende los catorce reactivos originales con autovalores arriba de 1, que en conjunto explican $59.6 \%$ de la varianza; las cargas factoriales de sus reactivos van de 0.817 a 0.640 . En la Tabla 2 se muestran la media, la desviación estándar y la carga factorial de cada uno de los reactivos finales.

Tabla 2. Estructura factorial, medias y desviación estándar por reactivo del HADS (Escala global, $\alpha=.94$; Varianza explicada $=59.6 \%)$.

\begin{tabular}{|c|c|c|c|c|}
\hline Núm. & Reactivos & $\mathbf{M}$ & D. E. & Carga factorial \\
\hline 1 & Me siento tenso(a) o nervioso(a). & 1.92 & 0.93 & 0.726 \\
\hline 2 & Todavía disfruto con lo que antes me gustaba. & 1.51 & 1.03 & 0.732 \\
\hline 3 & Tengo una sensación de miedo, como si algo horrible me fuera a suceder. & 1.89 & 1.02 & 0.788 \\
\hline 4 & Puedo reírme y ver el lado divertido de las cosas. & 1.39 & 1.06 & 0.792 \\
\hline 5 & Tengo mi mente llena de preocupaciones. & 1.84 & 1.03 & 0.810 \\
\hline 6 & Me siento alegre. & 1.67 & 1.00 & 0.776 \\
\hline 7 & Puedo estar sentado(a) tranquilamente y sentirme relajado(a). & 1.81 & 0.98 & 0.797 \\
\hline 8 & Me siento como si cada día fuera más lento. & 1.89 & 0.97 & 0.741 \\
\hline 9 & Tengo una sensación extraña, como de “aleteo” en el estómago. & 1.50 & 1.00 & 0.756 \\
\hline 10 & He perdido el interés en mi aspecto personal. & 1.60 & 1.20 & 0.817 \\
\hline 11 & Me siento inquieto(a), como si no pudiera parar de moverme. & 1.66 & 0.89 & 0.807 \\
\hline 12 & Me siento optimista respecto al futuro. & 1.40 & 1.08 & 0.640 \\
\hline 13 & Me asaltan sentimientos repentinos de pánico. & 1.75 & 1.08 & 0.814 \\
\hline 14 & Me divierto con un buen libro, la radio o un programa de televisión. & 1.50 & 1.20 & 0.793 \\
\hline
\end{tabular}

La confiabilidad de la escala ERCTA obtuvo un coeficiente alfa de Cronbach total de 0.930 y sus correlaciones internas fueron altas, por lo que se corrió un análisis factorial oblicuo de componentes principales. Este último análisis identificó un solo factor, equivalente al de la escala original, que explica $67.3 \%$ de la varianza total. La media total para la escala fue de $27.5 \pm 6.5$ y una varianza total de 41.8 , con cargas factoriales en sus ocho reactivos de entre 0.782 y 0.853 . El valor KMO fue de $0.934(p=0.001)$, confirmando que la muestra utilizada fue suficiente para este análisis (Tabla 3). La correlación entre las escalas HADS y ERCTA fue de $0.437(p=0.01)$. 
Tabla 3. Estructura factorial, medias y desviación estándar por reactivo del ERCTA.

\begin{tabular}{|c|l|c|c|c|}
\hline Núm. & \multicolumn{1}{|c|}{ Reactivos } & M & D. E. & $\begin{array}{c}\text { Carga } \\
\text { factorial }\end{array}$ \\
\hline 1 & ¿Considera usted que vive sometido a estrés? & 3.38 & 1.09 & 0.798 \\
\hline 2 & ¿Tiene usted un nivel de actividad? & 3.30 & 1.00 & 0.827 \\
\hline 3 & ¿Tiene usted una tendencia al perfeccionismo? & 3.43 & 0.97 & 0.830 \\
\hline 4 & Su deseo de llegar a los máximos niveles profesionales o sociales es: & 3.42 & 0.95 & 0.782 \\
\hline 5 & Su nivel de competitividad es: & 3.44 & 0.95 & 0.820 \\
\hline 6 & Su sensación de prisa y falta de tiempo es: & 3.55 & 1.01 & 0.853 \\
\hline 7 & Su necesidad de estar atento a su trabajo u ocupaciones es: & 3.51 & 0.99 & 0.826 \\
\hline 8 & El grado de dificultad para comunicar sus emociones es: & 3.48 & 0.93 & 0.826 \\
\hline
\end{tabular}

\section{DISCUSIÓN}

El presente estudio tuvo como propósito la adaptación cultural y la validación del HADS y el ERCTA en un grupo de 200 pacientes con cardiopatías. En relación al HADS, la consistencia interna y la varianza identificadas en este estudio son las más altas reportadas en las validaciones en población mexicana. Presentó una estructura unifactorial que, aunque no coincidió con la de dos factores de la versión original de Zigmond y Snaith (1983), concuerda con estudios que han identificado una sola dimensión (Nogueda et al., 2013; Razavi, Delvaux, Favacques y Robay, 1990; Reda, 2011), lo que sugiere que las diferencias que se pueden observar en el comportamiento de la escala y los constructos que evalúa son diferentes según la población clínica a la que se aplique el instrumento (Barriguete et al., 2017; Nogueda et al., 2013).

Al hallarse que los reactivos de ansiedad y depresión estuvieron fuertemente correlacionados y se agruparon en un factor, se propone que la estructura factorial del HADS en este grupo de pacientes se evalúe de forma global, lo que concuerda con la existencia de un factor de distrés general, como en los estudios previos de Razavi et al. (1990), Reda (2011) y Nogueda et al., (2013). Lo anterior puede indicar que los pacientes evalúan la sintomatología de ansiedad y depresión como un grupo de síntomas indistintos. En este sentido, Smith y Blumenthal (2011) describen tal sintomatología como un estado afectivo negativo, que es un rasgo más general, tipificado por las experiencias emocionales negativas, que engloba la ansiedad y la depresión, lo que se concuerda con lo reportado por Nogueda et al. (2013), quienes proponen que el puntaje total del HADS evalúa de forma global el distrés psicológico, y no la depresión y la ansiedad como entidades separadas y distintas (véase Tabla 4).

En relación con la estructura unifactorial del ERCTA obtenida en este estudio, los factores "estrés" y "ambición laboral" contrastan con los originales de tensión o estrés laboral y nivel de ambición profesional identificados en la versión original de Rodríguez et al. (1996). Aparecen aquí como un solo factor, lo que puede deberse a que la muestra de la versión original se integró con usuarios de centros de promoción de la salud, en contraste con la presente muestra de pacientes mexicanos con diagnóstico de enfermedad cardiovascular confirmada, tratamiento médico y en consulta ambulatoria, por lo que en la vida de estos pacientes el estrés puede estar implícito en las actividades laborales. Son asimismo diferentes en los componentes "presión competitiva" (competitive drive) e "impaciencia" (impatience) que, según Matthews (1982), son factores que se asocian con la aparición de trastornos cardíacos.

Por otra parte, ninguno de esos factores puede identificarse con la hostilidad, que se ha reportado como un factor asociado a los problemas cardiovasculares (Palmero et al., 2007; Smith y Blumenthal, 2011), aunque el factor único, denominado "estrés y ambición laboral", se halla conceptualmente relacionado. Tales diferencias se pueden encontrar dependiendo de la población a la que se apliquen los instrumentos de evaluación, y de ahí la importancia de reexaminar las propiedades psicométricas de todos ellos. 
Tabla 4. Comparación de propiedades psicométricas de las diferentes validaciones del HADS en población mexicana.

\begin{tabular}{|l|c|c|c|c|}
\hline \multicolumn{1}{|c|}{ Validaciones } & $\begin{array}{c}\text { Coeficiente } \\
\text { alfa de } \\
\text { Cronbach }\end{array}$ & $\begin{array}{c}\text { Varianza } \\
\text { explica- } \\
\text { da }\end{array}$ & Factores & $\begin{array}{c}\text { Número } \\
\text { total } \\
\text { de ítems }\end{array}$ \\
\hline Sujetos obesos (López et al., 2000). & 0.84 & - & $\begin{array}{c}\text { 1 Ansiedad } \\
\text { 2 Depresión }\end{array}$ & 14 \\
\hline $\begin{array}{l}\text { Mujeres en puerperio complicado, embarazo de alto riesgo, } \\
\text { cirugía general, ginecología u oncología (Villegas, 2004). }\end{array}$ & 0.78 & $44 \%$ & $\begin{array}{c}1 \text { Ansiedad } \\
\text { 2 Depresión }\end{array}$ & 14 \\
\hline Pacientes con cáncer de mama únicamente (Ornelas et al., 2011). & 0.85 & $51.79 \%$ & $\begin{array}{c}\text { 1 Ansiedad } \\
\text { 2 Depresión }\end{array}$ & 13 \\
\hline Pacientes con infección por VIH (Nogueda et al., 2013). & 0.90 & $54.65 \%$ & 1 Distrés & 13 \\
\hline Pacientes oncológicos (Galindo, Meneses et al. 2015). & 0.86 & $48.04 \%$ & $\begin{array}{c}\text { 1 Ansiedad } \\
\text { 2 Depresión }\end{array}$ & 12 \\
\hline $\begin{array}{l}\text { Cuidadores primarios informales de pacientes con cáncer } \\
\text { (Galindo et al., 2015). }\end{array}$ & 0.83 & $42.58 \%$ & $\begin{array}{c}\text { 1 Ansiedad } \\
\text { 2 Depresión }\end{array}$ & 14 \\
\hline Personas con trastorno de la conducta alimentaria (Barriguete et al., 2017). & 0.88 & $56.30 \%$ & $\begin{array}{c}\text { 1 Ansiedad } \\
\text { 2 Depresión }\end{array}$ & 12 \\
\hline Pacientes con enfermedades cardiovasculares (Herrera et al., 2017) & 0.94 & $67.30 \%$ & 1 Distrés & 14 \\
\hline
\end{tabular}

De acuerdo a los hallazgos de este estudio, se propone un factor general de conducta tipo A asociada al estrés en actividades laborales. Algunos autores (Everson, McKee y Lovallo, 1995; Laude, Girard, Consoli, Mounier-Vehier y Elghozi, 1997) han reportado que el patrón de conducta tipo A, con características que predisponen al estrés y a eventos estresantes, parece estar relacionado con mecanismos autonómicos y neuroendocrinos que se activan en dichas condiciones. La evaluación de la conducta tipo A en pacientes con cardiopatías es, pues, esencial debido a que puede ser un detonante de la enfermedad; así, al ser detectada, puede trabajarse sobre ella y evitar posibles recaídas.

En relación con la validez concurrente, las escalas HADS y ERCTA correlacionaron de manera positiva, estadísticamente significativa, con una magnitud baja, lo que respalda las asociaciones que se han identificado entre estas dos variables en diversos grupos de pacientes. En consecuencia, se propone continuar evaluando patologías especificas del área cardiovascular y hacer seguimientos a seis y doce meses después de la consulta con el propósito de determinar con mayor exactitud las patologías que se ven más afectadas por el distrés psicológico o conducta tipo A.

En conclusión, durante el curso de la enfermedad el equipo de salud debe evaluar si los pacientes tienen percepciones negativas o poco realistas de sí mismos, de su mundo y su futuro, y tratar de reestructurar los esquemas disfuncionales que pueden interferir con la percepción de su calidad de vida (Innamorati et al., 2014). A las emociones "negativas" se les ha asociado a un aumento de las tasas de muerte cardiovascular y de eventos cardiacos recurrentes (Smith y Blumenthal, 2011). La evaluación de la sintomatología en pacientes con cardiopatías es de gran importancia en virtud de que es la primera causa de muerte en el mundo, por lo que los síntomas reportados se han vuelto una variable crítica en la toma de decisiones respecto al tratamiento. El autorreporte de síntomas es asimismo fundamental para evaluar los efectos benéficos de los tratamientos farmacológicos, de su toxicidad y de la calidad de la supervivencia (Wang, Li y Wang, 2010).

Las propiedades psicométricas del Inventario de Ansiedad y Depresión y de la Escala de Retiro de Patrón de Conducta tipo A en una población de pacientes mexicanos con enfermedades cardiovasculares mustran una estructura unifactorial, lo que implica un conjunto de síntomas emocionales y conductuales negativos identificados en tales pacientes. Estos instrumentos, al ser breves, pueden ser útiles cuando se aplican en los ámbitos clínico y de investigación.

Entre las limitaciones de este trabajo se encuentra la falta de una entrevista clínica para confirmar los posibles casos de depresión o ansiedad como trastornos que evidencien las variables estu- 
diadas. Se sugiere, por ende, evaluar la estabilidad de ambos instrumentos a lo largo del tiempo, su sensibilidad al cambio en los procedimientos mé- dicos y su efectividad para identificar los efectos de las intervenciones psicológicas en este grupo de pacientes.

\section{REFERENCIAS}

Achiong, A., Achiong, F., Achiong, F. (2015). Prevención de la cardiopatía isquémica, un desafío de la atención primaria de salud. Revista Médica Electrónica, 37(2), 141- 53.

American Psychiatric Association (2014). Diagnostic and Statistical Manual of Mental Disorders V (DSM-R V). Washington, D.C.: APA

Barefoot, J., Larsen, S., Von der Leith, L. y Schroll, M. (1995). Hostility, incidence of acute myocardial infarction, and mortality in a sample of older Danish men and women. American Journal of Epidemiology; 142, 477-484.

Barriguete, J., Pérez, A., De la Vega, R., Barriguete, P. y Rojo, L. (2017). Validation of the Hospital Anxiety and Depression Scale in Mexican population with eating disorders. Revista Mexicana de Trastornos Alimentarios, 8, 123-130.

Bjelland, I., Dahl, A., Haug, T. y Neckelmann, D. (2002). The validit of the Hospital Anxiety and Depression Scale. An updated literature review. Journal of Psychosomatic Research, 52, 69-77.

Bortner, R.W. (1969). A short rating scale as a potential measure of pattern A behavior. Journal of Chronic Diseases, 22(2), 87-91.

Evans, P. (1991). Coronary heart disease. En M. Pitts y K. Philips (Dirs.): The psychology of health. An introduction (pp. 205224). London: Routledge.

Everson, S., Kauhanen, J., Kaplan, G., Goldberg, D., Julkunen, J., Tuomilehto J. y Salonen, J. (1997). Hostility and increased risk of mortality and acute myocardial infarction: the mediating role of behavioral risk factors. American Journal of Epidemiology, 46, 142-152.

Everson, S., McKee, B. y Lovallo, W. (1995). Effect of trait hostility on cardiovascular responses to harassment in young men. International Journal of Behavioral Medicine, 2, 172-91.

Frasure-Smith N., Lespérance F. y Talajic M. (1993). Depression following myocardial infarction: Impact on 6-month survival. Journal of the American Medical Association, 270(15), 1819-1825.

Friedman, M. (1996). Type A behavior: Its diagnosis and treatment. New York, NY: Plenum Press.

Friedman, M. y Rosenman, R.H. (1974). Type A behavior and your heart. New York, NY: Knopf.

Galindo, O., Benjet, C., Juárez, F., Rojas, E., Riveros, A., Aguilar P., J., Álvarez, M. y Alvarado, S. (2015). Propiedades psicométricas de la Escala Hospitalaria de Ansiedad y Depresión (HADS) para pacientes con cán $\neg$ cer en población mexicana. Salud Mental, 4(38), 253-258.

Galindo, O., Meneses, A., Herrera, A., Caballero M. y Aguilar, J. (2015). Escala Hospitalaria de Ansiedad y Depresión (HADS) en cuidadores primarios informales de pacientes con cáncer: propiedades psicométricas. Psicooncología, 12(2-3), 383-392.

Haynes, S., Levine, S.O.L., Scotch, N., Feinleib, M. y Kannel, W. (1978). The relationship of psychosocial factors to coronary heart disease in the Framingham study: I. Methods and risk factors. American Journal of Epidemiology, 107(5), $362-383$.

Herrera C., Deissy, C.A., G.A., Muñoz Z., D.A., Graillet M., O., Aranda A., G.E., Rojas D., F., Hernández M.E. y Nafissa, I. (2017). Impacto del estrés psicosocial en la salud. Neurobiología (Revista electrónica). Recuperado de www.uv.mx/eneurobiologia/vols/2017/17/17.html.

Instituto Nacional de Estadística y Geografía (2016). Tasa de morbilidad de los principales casos nuevos de enfermedades. México: INEGI.

Innamorati, M., Lester, D., Balsamo, M., Erbuto, D., Ricci, F., Amore, M. y Pompili, M. (2014). Factor validity of the Beck Hopelessness Scale in Italian medical patients. Journal of Psychopathology and Behavioral Assessmnt, $36(2), 300-307$.

Jenkins, C.D., Rosenman, R.H. y Zyzanski, S.J. (1979). Jenkins Activity Survey (Form C). San Antonio, TX: Psychological Corporation.

Kawachi, I., Sparrow, D., Spiro, A., Vokonas, P. y Weiss, S. (1996). A prospective study of anger and coronary heart disease: The Normative Aging Study. Circulation, 94: 2090-2095.

Koller, M., Aaronson, N., Blazeby, J., Bottomley, A., Dewolf, L., Fayers, P., y EORTC Quality of Life Group (2007). Translation procedures for standardized quality of life questionnaires: The European Organisation for Research and Treatment of Cancer (EORTC) approach. European Journal of Cancer, 43(12), 1810-1820.

Lane, D., Chong, A. y Lip, G. (2006). Intervenciones psicológicas para la depresión en la insuficiencia cardiaca (Revisión Cochrane traducida). Chichester, UK: John Wiley \& Sons, Ltd.

Laude, D., Girard, A., Consoli, S., Mounier-Vehier, C. y Elghozi, J. (1997). Anger expression and cardiovascular reactivity to mental stress: a spectral analysis approach. Clinical Exprimental Hypertension, 19(5-6), 901-911. 
Lichtman, J., Bigger, J., Blumenthal, J., Kaufman, P., Lesperance, F., Mark, D., Shep, D. y Tylor, B. (2008). Depression and coronary heart disease: Recommendations for screening referral and treatment: a science advisory. Circulation, 118, 1768-1775.

López, J., Vázquez, V., Arcila, D., Sierra, A., González, J. y Salin, R. (2000). Exactitud y utilidad diagnostica del Hospital Anxiety and Depression Scale (HAD) en una muestra de sujetos obesos mexicanos. México: Instituto Nacional de Ciencias Médicas y Nutricion Salvador Zubirán.

Matthews, K. (1982). Psychological perspectives on the type A behaviour pattern. Psychological Bulletin, 91, $293-323$.

Mittleman, M., Maclure, M., Sherwood, J., Mulry, R., Tofler, G., Jacobs., S. et al. (1995). Triggering of acute myocardial infarction onset by episodes of anger. Circulation, 92, 1720-1725.

Moser, D. (2007). "The rust of life": Impact of anxiety on cardiac patients. American Journal of Critical Care, 16(4), 361-369.

Muller, J., Abela, G., Nesto, R. y Tofler, G. (1994). Triggers, acute risk factors, and vulnerable plaques: the lexicon of a new frontier. Journal of the American College of Cardiology, 23, 809-813.

Nogueda, M., Pérez, B., Barrientos V., Robles, R. y Sierra, J. (2013). Escala de Ansiedad y Depresión Hospitalaria (HADS): validación en pacientes mexicanos con infección por VIH. Psicología Iberoamericana, 21(2), 29-37.

Organización Mundial de la Salud (2015). Enfermedades cardiovasculares. Ginebra: OMS. Recuperado de http://www.who.int/ mediacentre/fachtsheets/fs317/es/.

Ornelas M., R., Tufiño, M. y Sánchez-Sosa, J.J: (2011). Ansiedad y depresión en mujeres con cáncer de mama en radioterapia: prevalencia y factores asociados. Acta de Investigación Psicológica, 1(3), 401-414.

Palmero, F., Gómez, C., Guerrero, C., Capri, A., Diez, J. y Diago, J. (2007). Hostilidad psicofisiológica y salud cardiovascular. Avances en Psicología Latinoamericana, 25(1), 22-43.

Razavi, D., Delvaux, N., Farvacques, C. y Robaye, E. (1990). Screening for adjustment disorders and major depressive disorders in cancer in-patients. The British Journal of Psychiatry, 156(1), 79-83.

Reda, A. (2011). Reliability and validity of the Ethiopian version of the Hospital Anxiety and Depression Scale (HADS) in HIV infected patients. PLoS One, 6(1), e16049. doi: 10.1371/journal.pone.0016049. Recuperado de http://journals.plos.org/ plosone/article?id=10.1371/journal.pone.0016049.

Rodríguez S., C., Gil C., P. y Martínez, R. (1996). Presentación de la Escala Retiro de Patrón de Conducta Tipo A (ERCTA). Psicothema, 8(1), 207-213.

Roger, V., Lloyd-Jones, D., Adams, R., Brown, T. et al. (2010). Heart disease and stroke statistics. Circulation, 121(7), e46-e215.

Sistema Nacional de Información en Salud (s/f). Diez principales causas de mortalidad en hombres y mujeres por grupo de edad. México: SNIS. Recuperado de http://www.sinais.salud.gob.mx/mortalidad/index.html.

Smith, P. y Blumenthal, J. (2011). Aspectos psiquiátricos y conductuales de la enfermedad cardiovascular: epidemiología, mecanismos y tratamiento. Revista Española de Cardiología, 64(10), 924-933.

Tuckey, M., Dollard, M., Saebel, J. y Berry, N. (2010). Negative workplace behavior: Temporal associations with cardiovascular outcomes and psychological health problems in Australian police. Stress and Health, 26, 372-381.

Thombs, D. (2006). Introduction to addictive behaviors. New York: The Guilford Press.

Thombs, B., De Jonge, P., Coyne, J., Whooley, M., Frasure-Smith, N., Mitchell, A. et al. (2008). Depression screening and patient outcomes in cardiovascular care: a systematic review. Journal of the American Medical Association, 300, $2161-2171$.

Vázquez Ó., G., García A., M., Gómez Á., H., Del Rosario, M., Tinoco, C. y Ponce J., L.A. (2015). Escala Hospitalaria de Ansiedad y Depresión (HADS) en cuidadores primarios informales de pacientes con cáncer: propiedades psicométricas. Psicooncología, 12(2/3), 383.

Villegas, G. (2004). Reporte de experiencia profesional. Tesis de maestría en Psicología. México: UNAM.

Wang, J., Li, C. y Wang, E. (2010). Potential and flux landscapes quantify the stability and robustness of budding yeast cell cycle network. Proceedings of the Natural Sciences Academy, 107(18), 8195-8200.

Willich, S., Maclure, M., Mittleman, M., Arntz, H. y Muller, J. (1993). Sudden cardiac death. Support for a role of triggering in causation. Circulation, 87, 1442-1450.

Yohannes, A., Willgoss, T., Baldwin, R. y Connolly, M. (2010). Depression and anxiety in chronic heart failure and chronic obstructive pulmonary disease: prevalence, relevance, clinical implications, and management principles. International Journal of Geriatric Psychiatry, 25, 1209-1221.

Zigmond, A. y Snaith, R (1983). The Hospital Anxiety and Depression Scale. Acta Psychiatric Scandinavic, 67, $361-370$. 